\title{
Resource allocation in a generalized LTE air interface virtualization framework exploiting user behavior
}

\author{
Bo Fan ${ }^{1}$, Hui Tian ${ }^{1 *}$ and Xiao Yan²
}

\begin{abstract}
Wireless network virtualization (WNV) is a promising technique to solve the ossification of current networks. In this paper, a generalized Long Term Evolution (LTE) air interface virtualization framework is proposed where virtual operators (VOs) are enabled to share the physical resources owned by the infrastructure provider (InP). This user-centric feature provides VOs with the flexibility to manage their own virtualized networks according to different user traffic demand. Hence, we introduce the "Gini Coefficient" to quantitatively characterize the user traffic behavior among different VOs. In addition, we consider bandwidth-power allocation to optimize system energy efficiency (EE). The resource allocation problem is formulated as a mixed combinatorial and non-convex optimization problem, which is extremely difficult to solve. To reduce the computational complexity, we decouple the problem into two steps. First, for a given power allocation, we obtain the bandwidth allocation. Adopting bankruptcy game model and the well-known Shapley value, a heuristic bandwidth allocation algorithm is devised. Second, under the assumption of known bandwidth allocation, we transform the original optimization problem into an equivalent convex optimization problem and obtain the optimal solution via fractional programming. Through simulation, the results of user behavior and resource allocation are jointly analyzed. The user behavior is proved to be effective and the proposed resource allocation outperforms conventional schemes.
\end{abstract}

Keywords: LTE air interface virtualization, Resource allocation, User behavior

\section{Introduction}

Internet-dependent lifeway will greatly increase the diversity and density of communication demands in the nextgeneration mobile network (5G) [1]. This will lead to a burst of wireless traffic volume, which is anticipated to increase tenfold for the next decade [2]. However, today's ossified network operation greatly limits network capacity and efficiency. To cope with the situation, wireless network virtualization (WNV) draws worldwide attention for its potential to enhance flexibility, diversity, manageability, and energy efficiency for current networks [3].

The main idea of WNV is to enable differentiated services to run on common network infrastructure [3]. In $\mathrm{WNV}$, the traditional role of mobile network provider is

*Correspondence: tianhui@bupt.edu.cn

${ }^{1}$ State Key Laboratory of Networking and Switching Technology, Beijing University of Posts and Telecommunications, Beijing, China

Full list of author information is available at the end of the article separated into two parts. One is referred to as infrastructure provider (InP), who owns and supervises the overall physical network infrastructure. The other is virtual operator (VO) whose concentration is paid on offering on-demand services to their customers by purchasing resources from InP.

\subsection{Related work}

One primary research trend of WNV is Long Term Evolution (LTE) air interface virtualization, where multiple VOs share resource on the air interface [4]. Gudipati et al. [5] proposes logically abstracting multiple LTE eNodeB (evolved NodeB) as one virtual big base station. Hence, all the resource elements can be conceptually thought of as three-dimensional resource grid with time, frequency, and space. Li et al. [6] promotes replacing LTE eNodeBs with remote radio units (RRUs) in order to achieve complete virtualization. Thus the distributed control units can be replaced by a central controller, 
which maintains a global view of the radio access network (RAN). Yang et al. [7] refractors the LTE control plane as software-defined to enhance classified user traffic quality of service (QoS). The network performance is therefore improved due to the additional flexibility provided to user service.

Basically, the virtualization of the wireless resource on the air interface can be considered as a resource scheduling problem [8]. Existing researches mainly focus on bandwidth allocation. A contract-based bandwidth allocation algorithm is developed, where resources are allocated based on four types of pre-defined contracts $[9,10]$. Costa-Perez et al. [11] proposes virtualizing LTE eNodeB into different bandwidth slices. The virtualized slices are assigned to multiple mobile users according to their differentiated priority of service flows.

Recently, game theory has been applied in the context of WNV. Authors in [12] firstly introduce stochastic game to model the interaction between InP and the VOs. VOs dynamically compete for bandwidth resource from InP, and the allocation is determined when the bidding price is proved to reach a unique Nash equilibrium. To guarantee allocation fairness, bankruptcy game is proposed in [13] to share the limited LTE air interface bandwidth resource. Besides, [14] focuses on power resource allocation in LTE air interface virtualization, where a Vickrey-Clarke-Groves (VCG) auction is used to model InP as auctioneer selling power resource to multiple VOs.

\subsection{Main contributions}

Brilliant as above researches are, there are still several disadvantages. Firstly, since conventional network architecture no longer suits virtualization, the functionalities in operating virtualized network need to be re-defined. And there lacks a systematic virtualization framework for current LTE networks. Secondly, the user-centric feature in WNV is neglected. By purchasing resources from InP, VOs can operate their own virtualized networks. In other words, VOs can expand or shrink their markets and customize different types of services to satisfy whatever their users need to accomplish the communication task. Therefore, we believe that exploiting user behavior can improve the resource allocation efficiency.

To address above issues, the main contributions of our work can be summarized as follows:

- A generalized LTE air interface framework is proposed where data plane, control plane, and interface are explicitly defined. To decouple service from infrastructure, conventional LTE eNodeB is split into twofold components: data base station (BS) and signaling controller. Data BS is contained in the data plane, connected through gate-way to the Internet. The signaling controller, InP, along with the VO belong to the control plane. InP is in charge of the radio access resource via the interface with the signaling controller. VOs apply for resource from InP to serve the customers through the interface with InP.

- A tractable expression to quantitatively characterize user behavior is utilized to indicate the equilibrium/disequilibrium of VOs' user traffic rate demand, which lays foundation for designing the resource allocation strategies. To the best of our knowledge, this is the first time to introduce user behavior analysis to solve WNV problems.

- We consider energy efficiency (EE) optimization from a system perspective with bandwidth and power allocation. The problem is formulated as a mixed combinatorial and non-convex optimization problem and we decouple it into two steps. A bankruptcy game is adopted to model VOs as players and the bandwidth as the total estate. Using Shapley value, a heuristic bandwidth allocation algorithm is devised to optimize EE. Afterwards, the optimal power allocation is obtained via fractional programming.

- Different from existing works, in our simulation, user behavior is analyzed in joint with resource allocation results. In different cases, the change of the user behavior pattern is in line with the resource allocation results. This indicates that user behavior analysis can be a potential technique to be used in WNV to improve its flexibility and efficiency.

The paper is organized as follows. Section 2 and Section 3 present system model and problem formulation, respectively. Detailed resource allocation strategies are investigated in Section 4. Simulation results are analyzed in Section 5. Section 6 concludes the paper.

\section{System model}

The proposed virtualization model is in Fig. 1. In this section, we will explicitly describe the model in two aspects. And the user behavior definition is presented to characterize the service traffic proportion of different VOs in the system.

To make the paper more readable, we illustrate the major notations in Table 1. And our proceeding work is based on the following assumptions:

- Overall system physical resource block (PRBs, the minimum LTE bandwidth allocation unit, consisting of 12 sub-carriers) are ideally orthogonal so there exists no interference problem.

- Consider a heavily loaded hotspot area where the available resource is limited to meet the total traffic demand. 


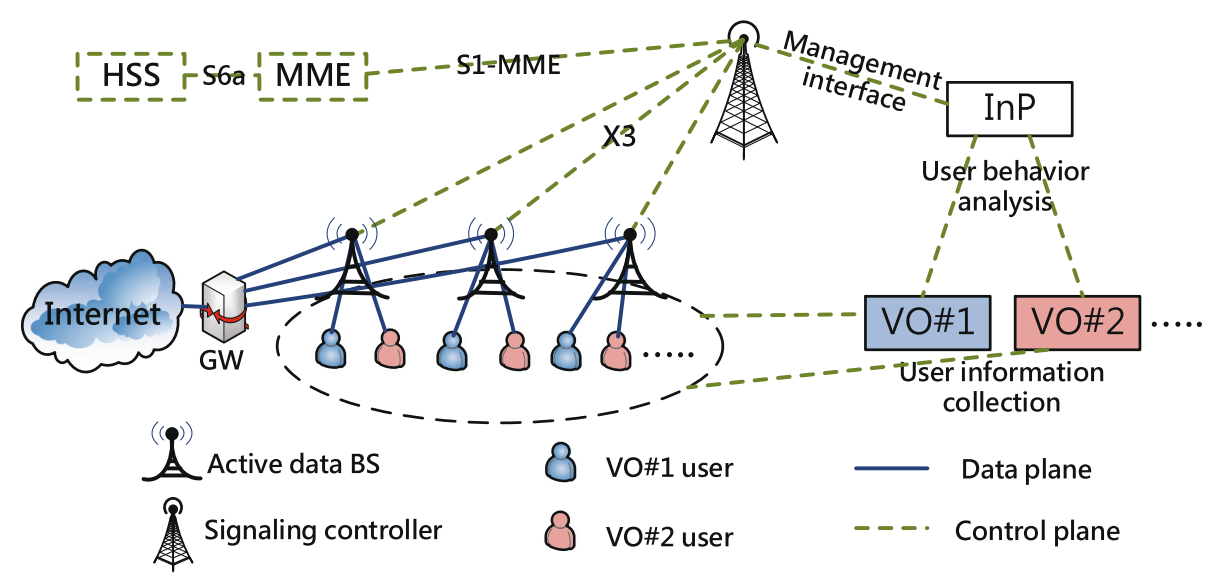

Fig. $1 \mathrm{LTE}$ air interface virtualization framework

Table 1 Basic notations used in the paper

\begin{tabular}{|c|c|}
\hline$v$ & VO index \\
\hline V & total VO number \\
\hline $\mathcal{S}$ & cooperative VO set \\
\hline $\mathcal{V}$ & total VO set \\
\hline$n$ & data BS index \\
\hline N & total data BS number \\
\hline k & PRB index \\
\hline$K_{v}$ & allocated PRBs number of $\mathrm{VO \#} \vee$ \\
\hline$m$ & user index \\
\hline$M_{v}$ & user number of $\mathrm{VO \#} \vee$ \\
\hline $\mathcal{M}_{V}$ & user set of VO\#v \\
\hline$d_{v}$ & average user traffic rate demand of $\mathrm{VO} \# \mathrm{v}$ \\
\hline$b_{v}$ & minimum PRB number to operate $\mathrm{VO \#} \vee$ \\
\hline$c_{v}$ & additional claimed PRB number of VO\# $\vee$ \\
\hline Y & estimated total PRB number to support the whole service area \\
\hline$\Phi$ & Shapley value \\
\hline y & characteristic function of coalition \\
\hline$K_{n}^{\max }$ & total PRB number of BS $n$ \\
\hline$P_{n}^{\max }$ & transmission power budget of BS $n$ \\
\hline$R_{k, n}^{m, v}$ & data rate on $\mathrm{PRB} k$ in $\mathrm{VO \#} \vee$ of $\mathrm{BS} n$ \\
\hline$g_{k, n}^{m, v}$ & user channel condition on PRB $k$ in VO\# $\vee$ of BS $n$ \\
\hline$a_{k, n}^{m, v}$ & PRB allocation indicator \\
\hline$p_{k, n}^{m, v}$ & transmit power on PRB $k$ in $\mathrm{VO \#} v$ of $\mathrm{BS} n$ \\
\hline$P_{c n}$ & circuit power of BS $n$ \\
\hline$\eta$ & power amplifier inefficiency \\
\hline$R_{\text {tot }}$ & total data rate \\
\hline$P_{\text {tot }}$ & total power assumption \\
\hline
\end{tabular}

\subsection{Virtualized air interface model}

As in Fig. 1, conventional LTE eNodeB functionalities are split into two components: signaling controller and data BS. Data BS belongs to the data-forwarding plane, connected through gate-way (GW) into the Internet. Its main task is to perform baseband processing and realize physical transmission tasks defined by the control plane. The main advantage of such function split is to improve network flexibility and energy efficiency. Energyconsuming data BSs are flexibly deployed and can be activated/deactivated based on different traffic conditions.

Signaling controller hosts control logic of RAN. Its main task is resource management on the air interface. For example, signaling controller can regulate data BSs activation/deactivation through interface X3 [15]. In night time, about $85 \%$ traffic reduction is predicted compared with peak-load time [16]. Therefore, in order to optimize network EE, redundant data BSs should be shut down. Also, sleeping BSs can be activated by signaling controller during busy hour.

In addition, signaling controller may also support part of the mobility management functions: when mobile users cross the coverage boundaries of data BSs or signaling controllers, handovers can be realized through the cooperation between signaling controllers and MME (linked via interface $\mathrm{S} 1$ ).

\subsection{InP-VO model}

In Fig. 1, a hot-spot area is serviced by several co-located data BSs. Consider one InP and multiple VOs, i.e., VO\#1 and $\mathrm{VO} \# 2$. VOs have distinct service objectives reflecting particular performance targets and constraints.

The task of VO is to ensure a satisfactory quality of experience (QoE) for their users. Since VOs have no direct access to physical network infrastructure, they may wish to buy wireless resource from the InP. Hence, VOs are 
required to decide how much radio resource to purchase from the InP. After obtaining the corresponding resource, VOs implement their own buffering strategy to satisfy users' needs (e.g., which user payloads to drop or which payloads to pass to the radio link layer). Therefore, VOs should be equipped with isolated memory space to carry out their own computation task without interfering with each other.

InP undertakes two tasks: (1) supervising the whole RAN and (2) supporting on-demand capacity requests from different VOs. On one hand, InP gathers VOs' user traffic information through the interface with different VOs. Based on the information, InP analyzes the global user traffic behavior according to a predefined contract or protocol. On the other hand, InP defines the wireless scheduling via management interface towards the signaling controller. The signaling controller, together with data BSs, perform resource allocation and physical transmission.

\subsection{User behavior curve}

A user behavior model for large-scale cellular users is introduced in [17-20]. The concept is based on Gini coefficient, an economic measure of statistical dispersion to evaluate the income distribution of a nation's residents [21]. It is an efficient index for assessment on regional income or wealth inequality. In this article, we extend the user behavior to more general cases and adopt it as a reference to describe VOs' traffic volume share in the overall system.

First, we sort all the VOs in ascending order of user traffic demand $d_{v}$ : $d_{1} \leq d_{2} \leq \cdots \leq d_{V}$. Thus, the user behavior curve based on [19] can be re-drawn as Fig. 2 .

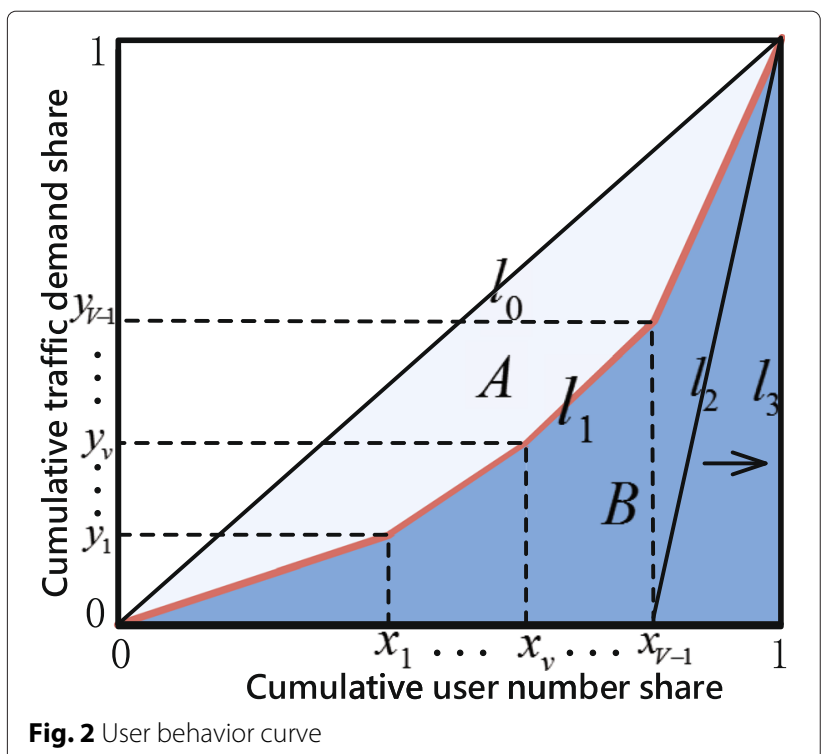

$X$-axis represents cumulative user number proportion from $\mathrm{VO} \# 1$ to $\mathrm{VO} \# V$, ranging from 0 to $1 . Y$-axis represents cumulative traffic demand proportion from $\mathrm{VO} \# 1$ to $\mathrm{VO} \# V$, ranging from 0 to 1 . In this way, $l_{1}$ in Fig. 2 forms the general $\mathrm{VO}$ user behavior curve. Each segment of $l_{1}$ stands for a certain $\mathrm{VO}$, and its slope equals to respective VO's traffic demand $d_{\nu}$. Therefore, the coordinate of fold point $\left(x_{v}, y_{v}\right)$ in $l_{1}$ can be calculated as follows:

$$
x_{v}=\frac{\sum_{i=1}^{v} M_{i}}{\sum_{i=1}^{V} M_{i}}, y_{v}=\frac{\sum_{i=1}^{v} M_{i} d_{i}}{\sum_{i=1}^{V} M_{i} d_{i}}
$$

where $v=1,2, \cdots, V$. Without loss of generality, let us define $m_{0}=0, d_{0}=0$, and the coordinate can be modified as:

$$
x_{v}=\frac{\sum_{i=0}^{v} M_{i}}{\sum_{i=0}^{V} M_{i}}, y_{v}=\frac{\sum_{i=0}^{v} M_{i} d_{i}}{\sum_{i=0}^{V} M_{i} d_{i}}
$$

where $x_{0}=y_{0}=0$.

Furthermore, in order to quantitatively depict the user behavior, we further introduce the user behavior coefficient concept to our model. As defined in [19], user behavior coefficient $h$ is defined as the ratio between the area " $\mathrm{A}$ " over the total area "A" plus "B". Area A is enclosed by curve $l_{0}$ and $l_{1}$. Area $\mathrm{B}$ is enclosed by curves $l_{1}$ and $l_{3}$ as well as $X$-axis. Since area B can be regarded as the sum of a triangle and several trapeziums, the area of $B$ can be calculated by adding up all the sub-graphs. Herein, we derive the expression of area $\mathrm{B}$ as follows:

$$
B=\sum_{v=1}^{V} \frac{\left(y_{v}+y_{v-1}\right)\left(x_{v}-x_{v-1}\right)}{2}
$$

Substituting Eq. (2) into (3), we can obtain:

$$
B=\frac{2 \sum_{v=1}^{V} \sum_{i=0}^{v-1} M_{i} M_{v} d_{i}+\sum_{v=1}^{V} M_{\nu}^{2} d_{v}}{2\left(\sum_{i=0}^{V} M_{i} d_{i}\right)\left(\sum_{i=0}^{V} M_{i}\right)}
$$

Next, according to the user behavior coefficient definition, $h$ can be calculated in relation to Eq. (4) as follows:

$$
h=\frac{A}{A+B}=\frac{(A+B)-B}{(A+B)}=1-2 B
$$

For better understanding user behavior coefficient $h$, we discuss it under two extreme cases:

- First, consider the case where users of each VO generate equal traffic rate demand, i.e., $d_{1}=d_{2}=\cdots=d_{V}$. Thus, Eq. (4) can be transformed as:

$$
B=\frac{2 \sum_{v=1}^{V} \sum_{i=0}^{v-1} M_{i} M_{v}+\sum_{v=1}^{V} M_{v}^{2}}{2\left(\sum_{i=0}^{V} M_{i}\right)^{2}}
$$


According to multinomial theorem [22], we have:

$$
\begin{aligned}
\left(\sum_{i=0}^{V} M_{i}\right)^{2} & =\sum_{i=0}^{V} M_{i}^{2}+2 \sum_{j=1}^{V} \sum_{i=0}^{j-1} M_{i} M_{j} \\
& =m_{0}^{2}+\sum_{i=1}^{V} M_{i}^{2}+2 \sum_{j=1}^{V} \sum_{i=0}^{j-1} M_{i} M_{j} \\
& =\sum_{v=1}^{V} M_{v}^{2}+2 \sum_{v=1}^{V} \sum_{i=0}^{v-1} M_{i} M_{v}
\end{aligned}
$$

Substituting (7) into (5) and (6), we can obtain $B=\frac{1}{2}, h=0$, which means the traffic demands are equivalent over the whole service area across various VOs (equilibrium). This way, the user behavior is completely equality and user behavior curve $l_{1}$ converges to curve $l_{0}$ in Fig. 2.

- In the second case, the traffic amounts demanded by multiple VOs are exceedingly disequilibrium. This may correspond to the situation that all the traffic demand are generated by one dominated $\mathrm{VO}$ while other VOs generate few amounts of traffic (called as "oligopoly" in economics). In this situation, we have $d_{i} \rightarrow 0, \forall i=1,2 \cdots, V-1$. So by observing Eq. (4) we find: $\sum_{v=1}^{V} \sum_{i=0}^{v-1} M_{i} M_{v} \rightarrow 0, \sum_{v=1}^{V} M_{v}^{2} d_{v} \rightarrow$ $M_{V}^{2} d_{V}$ and $\left(\sum_{i=0}^{V} M_{i} d_{i}\right)\left(\sum_{i=0}^{V} M_{i}\right) \rightarrow M_{V} d_{V}$ $\sum_{i=0}^{V} M_{i}$. Finally, we have: $B \rightarrow \frac{M_{V}}{2 \sum_{i=0}^{V} M_{i}}, h \rightarrow$ $1-\frac{M_{V}}{\sum_{i=0}^{V} M_{i}}$. This way, the user behavior is completely inequality and user behavior c urve $l_{1}$ converges to curve $l_{2}$ in Fig. 2. To take a step further, assuming that the user number of $\mathrm{VO} \# V$ is much less than the total number, i.e., $M_{V}<<\sum_{i=1}^{V} M_{i}$, so $\frac{M_{V}}{\sum_{i=0}^{V} M_{i}} \rightarrow 0$. In such condition curve, $l_{2}$ approaches to curve $l_{3}$ and $B \rightarrow 0, h \rightarrow 1$.

In conclusion, the value of user behavior coefficient generally lies in the interval $[0,1]$. In practice, however, both extreme values are barely reached. On the one hand, a low user behavior coefficient indicates the traffic demand share across all the VOs follows rather even distribution, with 0 corresponding to complete equilibrium. On the other hand, high user behavior coefficient indicates the traffic demand share of different VOs follows uneven distribution, with one corresponding to complete convergence (i.e., the total traffic demands are requested by few users of the dominated $\mathrm{VO}$ ).

\section{Problem formulation}

Our focus is on the resource allocation problem of $\operatorname{InP}$ to different VOs, and EE metric is adopted to evaluate the system performance. Given the channel state $g_{k, n}^{m, v}$, the transmitted data rate on $\mathrm{PRB}(k, n)$ (PRB $k$ of data BS $n$ ) of user $(m, v)$ (user $m$ of $\mathrm{VO} v$ ) can be calculated by:

$$
R_{k, n}^{m, v}=W \log _{2}\left(1+\frac{a_{k, n}^{m, v} g_{k, n}^{m, v} p_{k, n}^{m, v}}{\sigma^{2}}\right)
$$

where $\sigma^{2}$ and $W$ denote additive white Gaussian noise power and PRB bandwidth. $a_{k, n}^{m, v}$ is the PRB allocation indicator. $a_{k, n}^{m, v}=1$ denotes that PRB $(k, n)$ is allocated to user $(m, v)$; otherwise, $a_{k, n}^{m, v}=0$. The total amount of system-transmitted data rate $R_{\text {tot }}$ during one scheduling period can be calculated as: $R_{\text {tot }}=\sum_{v=1}^{V} \sum_{m=1}^{M_{v}} R_{k, n}^{m, v}=$ $\sum_{n=1}^{N} \sum_{k=1}^{K_{n}^{\max }} R_{k, n}^{m, v}$. Hence, the overall system power consumption can be expressed as:

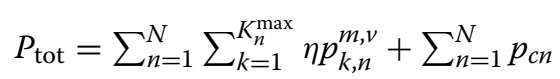

where $\eta$ is the power amplifier inefficiency. $p_{c n}$ denotes the circuit power of the data BS. The classic EE metric, i.e., the number of delivered bits per energy unit, is adopted in this paper [23]. Hence, the optimization problem for maximizing the system EE can be written as:

$$
\begin{aligned}
& \max _{\mathbf{A}, \mathbf{P}} \frac{R_{\text {tot }}(\mathbf{A}, \mathbf{P})}{P_{\text {tot }}(\mathbf{A}, \mathbf{P})} \\
& \text { s.t. } \\
& \text { C1 }: a_{k, n}^{m, v} \in\{0,1\}, \forall k, n, m, v \\
& \text { C2 : } \sum_{v=1}^{V} \sum_{m=1}^{M_{v}} a_{k, n}^{m, v}=1, \forall k, n \\
& \text { C3 : } \sum_{m=1}^{M_{v}} a_{k, n}^{m, v} \geq b_{v}, \forall k, n, v \\
& \text { C4 : } p_{k, n}^{m, v} \geq 0, \forall k, n, m, v \\
& \text { C5 }: \sum_{k=1}^{K_{n}^{\max }} p_{k, n}^{m, v} \leq P_{n}^{\max }, \forall n, m, v
\end{aligned}
$$

where $\mathbf{A}$ with element $a_{k, n}^{m, v}$ and $\mathbf{P}$ with element $p_{k, n}^{m, v}$ are the PRB allocation vector and the power allocation vector, respectively. $\mathrm{C} 1-\mathrm{C} 3$ are PRB allocation constraints. $\mathrm{C} 2$ means that one PRB can be only allocated to one user at most. $\mathrm{C} 3$ is used to guarantee the minimum bandwidth (PRB number) requirement of each VO. C4-C5 are the power allocation constraints, and $P_{n}^{\max }$ is the maximum transmit power of BS $n$.

The resource allocation problem in (10) is a mixed combinatorial and nonconvex optimization problem. The combinatorial nature comes from the PRB allocation constraints $\mathrm{C} 1$ and $\mathrm{C} 2$. The nonconvexity feature is caused by the fractional form of the objective function. Besides, the variables are mixed integer. Hence, the problem is very difficult to solve. Therefore, we decouple the resource allocation problem into two sub-problems: PRB (bandwidth) allocation for a given power allocation and power allocation for a given PRB allocation.

\section{Resource allocation strategy}

In this section, we first devise a heuristic PRB allocation algorithm using bankruptcy game and Shapley value. Second, power allocation problem is solved by fractional programming. 


\subsection{PRB allocation algorithm}

First, let us assume that power allocation is known, and sub-problem 1 can be defined by extracting constraints C1-C3 from (10):

$$
\begin{array}{r}
\max _{\mathbf{A}} \frac{R_{\text {tot }}(\mathbf{A})}{P_{\text {tot }}(\mathbf{A})} \\
\text { s.t. } \mathrm{C} 1, \mathrm{C} 2, \mathrm{C} 3
\end{array}
$$

The problem is a nonlinear integer programming under constraints. Conventional solutions include branch-andbound method and intelligent algorithm. For both methods, the computation complexity is rather high, especially when multiple constraints exist. In addition, one major concern of the problem is to guarantee allocation fairness, which is neglected in conventional solutions. Because the rate demands differentiate from user to user, allocation according to the demands efficiently optimizes the resource utilization.

In our work, bankruptcy game is introduced to solve the problem. VOs are modeled as bankrupt players, and total PRBs are modeled as overall estate. The reasons are as follows. First, bankruptcy defines a model where the estate is insufficient to satisfy the demand of all players [24]. Since the PRBs owned by the InP are limited, the total traffic demand should exceed the rate that the PRBs can provide (according to assumption 2). Second, as a cooperative game, bankruptcy game enables VOs to cooperatively share the PRB resource. Third, to guarantee the allocation fairness, Shapley value is adopted as a solution to the bankruptcy game [25].

In order to maintain the virtual network operated, each $\mathrm{VO}$ is likely to reserve a minimum number of PRBs $b_{v}$, where $\sum_{v=1}^{V} b_{v} \leq \sum_{n=1}^{N} K_{n}^{\max }$. In addition, each VO should claim additional PRBs $c_{v}$ to satisfy its customers' traffic needs as much as possible. This establishes competition among different VOs. Therefore, to guarantee fairness, the allocation strategy should be centrally handled by the InP based on the instantaneous traffic flow demand reported from VOs.

The InP firstly makes an assessment on the total PRB number $Y$ to support the whole service area. This can be realized by combining the most recent traffic demand information and average channel states in previous transmit time intervals (TTIs). We recommend that the InP may assume a rather deteriorated channel condition well below the average value, so that $Y>\sum_{n=1}^{N} K_{n}^{\max }-$ $\sum_{v=1}^{V} b_{v}$. Then, the additional claimed PRBs can be calculated through the following formulas:

$$
\begin{aligned}
& c_{v}=\left(\frac{M_{v} d_{v}}{\sum_{i=1}^{V} M_{i} d_{i}}\right) *(Y-V)+1, \forall v \\
& \sum_{v=1}^{V} c_{v}=Y
\end{aligned}
$$

In the bankruptcy game, VOs cooperatively share the physical resource and form coalition with each other. When a coalition of VOs cooperates, a certain overall payoff is obtained from the cooperation. Shapley value calculates the contribution or the relative importance that each player makes to the total payoff. First, let us define coalition $\mathcal{S}$ as a subset of $\mathcal{N}$ (i.e., $\mathcal{S} \subseteq \mathcal{N}$ ). For example, $\mathcal{S}=\{1,2\}$ means VO\#1 and VO\#2 cooperatively apply for the PRB resource in the bankruptcy game. Here, we define the cooperation payoff $(y$ in respect of $\mathcal{S}$ ) as [24]:

$$
y(S)=\left[\sum_{n=1}^{N} K_{n}^{\max }-\sum_{v=1}^{V} b_{v}-\sum_{v \notin S} c_{v}\right]^{+}
$$

In total, there are possibly $2^{N}$ coalitions and for each possible coalition, $y$ takes a particular value. For example, $y(\emptyset)=0$ means no VO cooperates in the game, while $y(N)=\sum_{n=1}^{N} K_{n}^{\max }-\sum_{v=1}^{V} b_{v}$ means all VOs cooperate. Using $y(S)$, Shapley value can be calculated as follows [25]:

$$
\Phi_{\nu}=\sum_{\mathcal{S} \subseteq \mathcal{N}, v \in \mathcal{S}}\left(\frac{(|\mathcal{S}|-1) !(N-|\mathcal{S}|) !}{N !}(y(\mathcal{S})-y(\mathcal{S}-\{v\}))\right)
$$

where $|\cdot|$ represents set's cardinality. The Shapley value is expressed as a weighted sum of the cooperation payoff. $y(\mathcal{S})-y(\mathcal{S}-\{v\})$ calculates the incremental payoff that VO $v$ owes to coalition $\mathcal{S}$. $\frac{(|\mathcal{S}|-1) !(N-|\mathcal{S}|) !}{N !}$ is a weighting factor that assigns equal share of the generated payoff to each coalition of interest. Shapley value calculates the contribution or the relative importance that each player (VO) makes to the total payoff. Thus, the optimal allocation PRB number $K_{v}$ can be calculated as follows:

$$
\begin{aligned}
& K_{v}=\left[b_{v}+\Phi_{v}\right], \forall v \\
& \sum_{v=1}^{V} K_{v}=\sum_{n=1}^{N} K_{n}^{\max }
\end{aligned}
$$

where symbol $[x]$ denotes rounding to the nearest integer of $x . b_{v}$ is the the minimum PRB number to maintain a certain VO network operated and $\Phi_{v}$ is the additional allocated PRB number calculated based on VO's traffic demand. (15b) means that the total allocated PRB number should meet the maximum PRB number constraint.

The PRB allocation algorithm is presented in Algorithm 1. In each iteration, PRB is allocated to VO user with the aim of maximizing energy efficiency. Operator filter() is defined as: filter $\left(\mathcal{M}_{v}, K_{v}\right)=\emptyset$ if and only if $K_{v}=0$; otherwise, filter $\left(\mathcal{M}_{v}, K_{v}\right)=\mathcal{M}_{v}$. filter() is used to guarantee $\mathrm{VO} v$ quitting the allocation when its allocated PRB number exceeds $K_{v}$. 


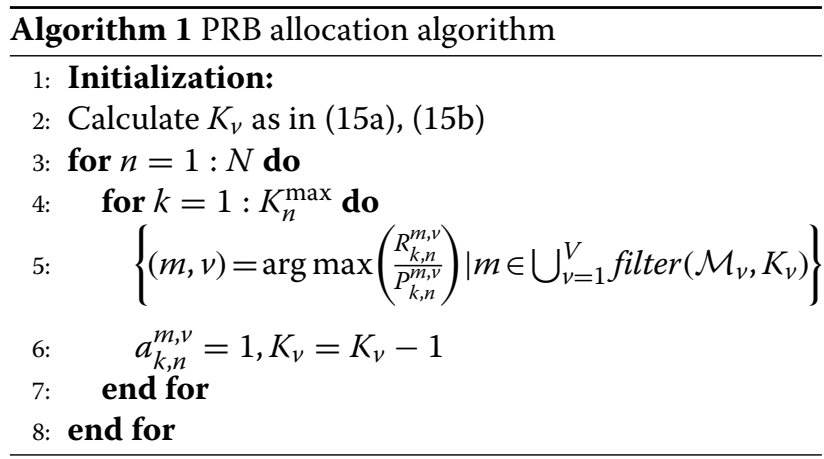

\subsection{Power allocation}

Assuming the PRB allocation is known, the original problem (10) is decoupled to a power allocation problem. To eliminate the non-convexity of the power allocation problem, we transform it into an equivalent convex optimization. And the optimal power allocation is obtained by Lagrange dual approach.

\subsubsection{Problem transformation}

Extracting constraints C4 and C5 from (10), sub-problem 2 can be written as:

$$
\begin{gathered}
\max _{\mathbf{P}} \frac{R_{\text {tot }}(\mathbf{P})}{P_{\text {tot }}(\mathbf{P})} \\
\text { s.t. C4, C5 }
\end{gathered}
$$

where (16) is non-convex due to the fractional form of the objective function. To make the problem more tractable, several transformations are required to eliminate the nonconvexity. Hence, let $q=\frac{R_{\text {tot }}}{P_{\text {tot }}}$, then we have the following theorem [26].

\section{Theorem 1.}

$$
\begin{aligned}
& q^{*}=R_{\text {tot }}\left(\mathbf{P}^{*}\right) / P_{\text {tot }}\left(\mathbf{P}^{*}\right)=\max \left\{R_{\text {tot }}(\mathbf{P}) / P_{\text {tot }}(\mathbf{P})\right\} \\
& \text { if, and only if, } \\
& \mathrm{F}\left(q^{*}\right)=\mathrm{F}\left(q^{*}, \mathbf{P}^{*}\right)=\max \left\{R_{\text {tot }}(\mathbf{P})-q^{*} P_{\text {tot }}(\mathbf{P})\right\}=0
\end{aligned}
$$

Theorem 1 is proved in Appendix 1. From Theorem 1, we know that for an optimization problem with a fractional form objective function, there exists an equivalent objective function in subtractive form. Thus, the original optimization problem in (16) can be reshaped into following formations:

$$
\begin{aligned}
& \max _{\mathbf{P}} R_{\text {tot }}(\mathbf{P})-q P_{\text {tot }}(\mathbf{P}) \\
& \text { s.t. } \mathrm{C} 4, \mathrm{C} 5
\end{aligned}
$$

where it is easy to verify that the objective is a concave function with respect to $p_{k}^{(v, n)}$. Hence, we can first solve (17) and then solve (16) by adopting iterative algorithms as given in Algorithm 2.
Algorithm 2 iteratively approximates the optimal solution of (17) towards a minimum threshold $\varepsilon(\varepsilon>0)$ and finally achieves optimal EE $q$ with the termination condition satisfied. We give the convergence proof of Algorithm 2 in Appendix 2. The algorithm converges to the optimal EE with a superlinear convergence rate [27].

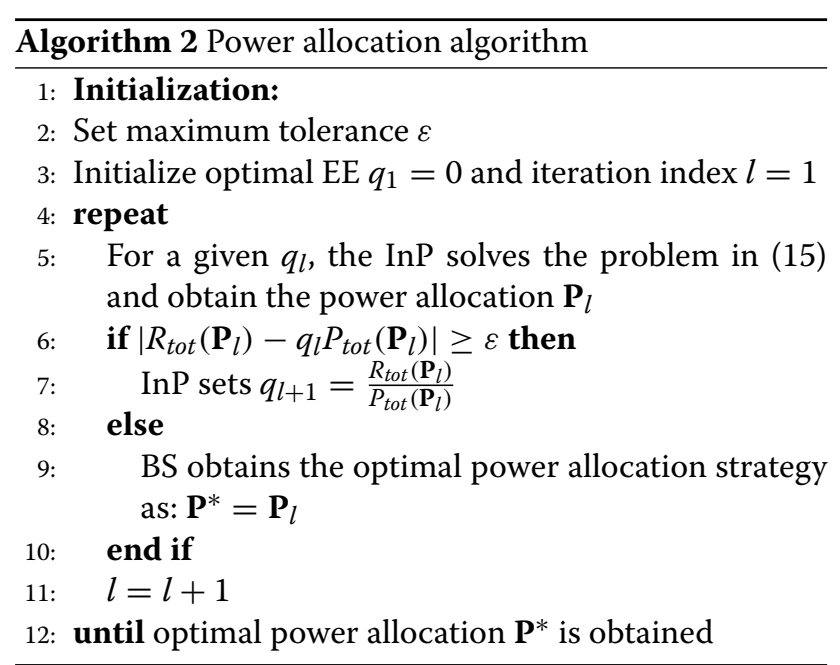

\subsubsection{Power allocation for transformed problem}

The optimization problem in (17) is a concave optimization problem. As proved in [28], the duality gap equals to zero under some mild conditions (strong duality). Therefore, we can use Lagrange duality method to solve problem (17). The Lagrange function can be derived as follows:

$$
\begin{aligned}
L\left(p_{k, n}^{m, v}, \lambda_{n}\right)= & \sum_{n=1}^{N} \sum_{k=1}^{K_{n}^{\max }} R_{k, n}^{m, v} \\
& -q\left(\sum_{n=1}^{N} \sum_{k=1}^{K_{n}^{\max }} \eta p_{k, n}^{m, v}+\sum_{n=1}^{N} P_{c n}\right) \\
& -\sum_{n=1}^{N} \lambda_{n}\left(\sum_{k=1}^{K_{n}^{\max }} p_{k, n}^{m, v}-P_{n}^{\max }\right)
\end{aligned}
$$

where $\lambda_{n} \geq 0(n=1,2, \cdots N)$ are the Lagrangian multipliers. Thus, the dual problem of (17) can be expressed as:

$$
\min _{\lambda_{n} \geq 0} \max _{p_{k, n}^{m, v} \geq 0} L\left(p_{k, n}^{m, v}, \lambda_{n}\right)
$$

Therefore, we can solve (19) by decomposing it into two layers. Layer 1: solve the maximization Lagrange problem with respect to $p_{k, n}^{m, v}$ under a fix set of Lagrange multipliers. Layer 2: obtain $\lambda_{n}$ by minimizing the Lagrange problem. 
1) Solution for layer 1: By dual decomposition, we first solve the following Layer 1 sub-problem:

$$
\max _{p_{k, n}^{m, v} \geq 0} L\left(p_{k, n}^{m, v}, \lambda_{n}\right)
$$

with a given parameter $q$ and a fixed set of Lagrange multipliers $\lambda_{n}$. Using standard KKT condition $\frac{\partial L\left(p_{k, n}^{m, v}, \lambda_{n}\right)}{\partial p_{k, n}^{m, v}}=0$, power allocation policy is obtained as:

$$
p_{k, n}^{m, v}=\left[\frac{W}{\left(q \eta+\lambda_{n}\right) \ln 2}-A_{k, n}^{m, v}\right]^{+}
$$

where $A_{k, n}^{m, v}=\frac{\sigma^{2}}{g_{k, n}^{m, v}}$.

2) Solution for layer 2: by substituting (21) into (19), layer 2 sub-problem can be described as follows:

$$
\min _{\lambda_{n} \geq 0} f\left(\lambda_{n}\right)
$$

where

$$
\begin{aligned}
& f\left(\lambda_{n}\right)=\sum_{n=1}^{N} \sum_{k=1}^{K_{n}^{\max }} \frac{W}{A_{k, n}^{m, v}\left(q \eta+\lambda_{n}\right) \ln 2} \\
& +\sum_{n=1}^{N} \sum_{k=1}^{K_{n}^{\max }} A_{k, n}^{m, v} \lambda_{n}+\sum_{n=1}^{N} \lambda_{n} P_{n}^{\max } \\
& +q \eta \sum_{n=1}^{N} \sum_{k=1}^{K_{n}^{\max }} A_{k, n}^{m, v}-q \sum_{n=1}^{N} P_{c n}
\end{aligned}
$$

Since $f\left(\lambda_{n}\right)$ is differentiable with respect to $\lambda_{n}$, we derive its second derivative form as follows:

$$
f^{\prime \prime}\left(\lambda_{n}\right)=\frac{K_{n}^{\max }}{\left(q \eta+\lambda_{n}\right)^{2} \ln 2}
$$

where it can be obviously observed that $f^{\prime \prime}\left(\lambda_{n}\right)>0$. According to [28], we know that $f\left(\lambda_{n}\right)$ is a convex function of $\lambda_{n}$. Therefore, we can obtain the optimal solution below:

$$
\lambda_{n}{ }^{*}=\left[\frac{W K_{n}^{\max }}{\left(\sum_{k=1}^{K_{n}^{\max }} A_{k, n}^{m, v}+P_{n}^{\max }\right) \ln 2}-q \eta\right]^{+}
$$

Thus, deriving (25) into (21), we can solve problem (17).

\section{Simulation and analysis}

In our paper, a hot-spot region is simulated under the proposed LTE air interface virtualization framework. The area is covered with three data BSs, shared by three VOs (i.e., VO\#1, VO\#2, and VO\#3). Mobile users are uniformly distributed in the area with two types of traffic configuration: video and VoIP. Simulation details are given in Table 2 .

In addition, "legacy" LTE network without virtualization is introduced as a benchmark to compare with the proposed virtualization architecture. In legacy LTE network setup, the network operators own their individual physical infrastructure and frequency band and operate isolated
Table 2 Simulation configuration

\begin{tabular}{ll}
\hline Parameter & Configuration \\
\hline Simulation area & Circular, $R=750[\mathrm{~m}]$ \\
& Coordinate of circle center $(0,0)$ \\
Data BS configuration & 3 data BS, 10 MHz bandwidth each \\
& $($ corresponds to about 50 PRBs) \\
Data BS coordinates & $(0,10)(-5,8.66)(5,8.66)$ \\
Data BS power & $P_{n}^{\max }=43 \mathrm{dBm}, P_{\text {circuit }}=25 \mathrm{dBm}$ \\
Path loss model & $128.1+37.6$ log 10(d) dB, d in km [29] \\
Slow fading model & Lognormal with zero mean value \\
& Standard deviation $=8 \mathrm{~dB}$ \\
Fast fading model & Correlation distance $=50 \mathrm{~m}$ \\
Modulation schemes & Jakes model \\
Link-2-System interface & QPSK, 16 QAM, 64 QAM \\
Video traffic bit rate & Effective exponential SINR mapping [30] \\
VolP traffic bit rate & 242 kbps [13] \\
\hline
\end{tabular}

from each other. In our simulation, each operator manages one of the three data BSs such that the resource on the air interface cannot be shared. To evaluate the proposed resource allocation strategy, three different cases are simulated, as shown in Table 3.

In case 1, 90 total users are equally distributed among the three operators. User traffic type is video streaming. According to user behavior definition in Eqs. (1)-(5), we calculate the user behavior coefficient as $h=0$, which means that the traffic demands generated by VOs are totally equilibrium. The allocated PRB number in virtualized and legacy network are compared in Fig. 3. In legacy setup, each operator is assigned with a fixed number of 50 PRBs via its operated BS. In virtualized setup, since different VOs generate equal traffic proportion, the total 150 PRBs are uniformly allocated (50 PRBs each VO).

In case 2, we deploy $40 \mathrm{VoIP}$ users to VO\#1, 20 video users to $\mathrm{VO} \# 2$, and 30 users to $\mathrm{VO} \# 3$. Users in operator 3 enjoy a mixed traffic of both video and VoIP. Based on

Table 3 Simulation case

\begin{tabular}{ll}
\hline Virtual operators configuration: & VO\#1: 30 video users \\
Case 1 & VO\#2: 30 video users \\
& VO\#1: 30 video users \\
Virtual operators configuration: & VO\#1: 40 VolP users \\
Case 2 & VO\#2: 20 video users \\
& VO\#2: 30 video+VoIP users \\
Virtual operators configuration: & VO\#1: 20 VolP users \\
Case 3 & VO\#2: 50 VolP users \\
& VO\#3: 20 video users \\
\hline
\end{tabular}




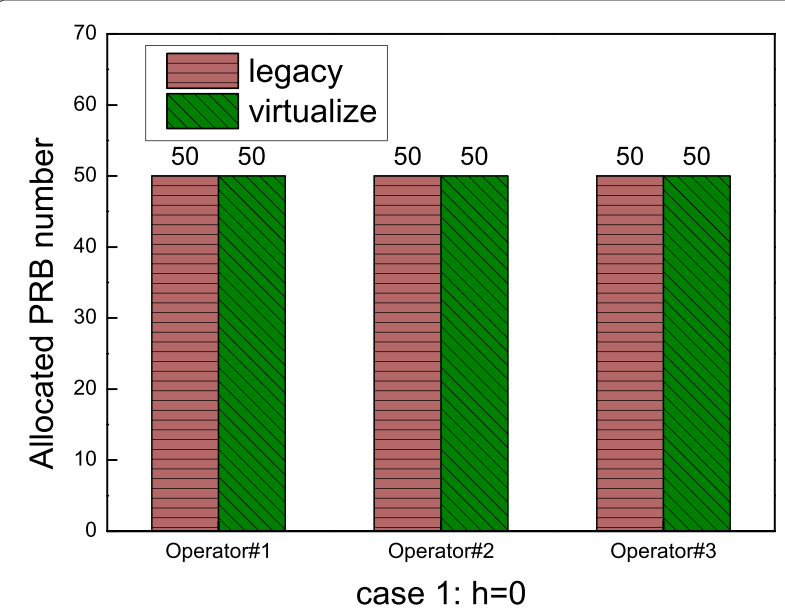

Fig. 3 Operator PRB allocation of case 1

Tables 2 and 3, we can approximately estimate the total rate demand relationship as: VO\#1< VO\#2< VO\#3. Similiarly, we calculate behavior coefficient as $h=0.42$. Figure 4 compares the PRB allocation of case 2. Legacy network demonstrates stable PRB allocation while the PRBs are allocated in relation to the traffic demand information of different VOs.

In case $3, \mathrm{VO} \# 1, \mathrm{VO} \# 2$, and $\mathrm{VO} \# 3$ are burdened with 20 VoIP users, 50 VoIP users, and 20 video users respectively. We can estimate that $\mathrm{VO} \# 3$ accounts for about $90 \%$ of the total traffic volume. In this disequilibrium case, the user behavior coefficient is calculated as $h=0.67$. The PRB allocation in Fig. 5 indicates that VO\#3 is allocated 116 out of 150 total PRBs. while the rest PRBs are shared by the other two VOs with lower traffic burdens.

In summary, the above three simulation cases reflect different scenarios with different VOs' service objectives

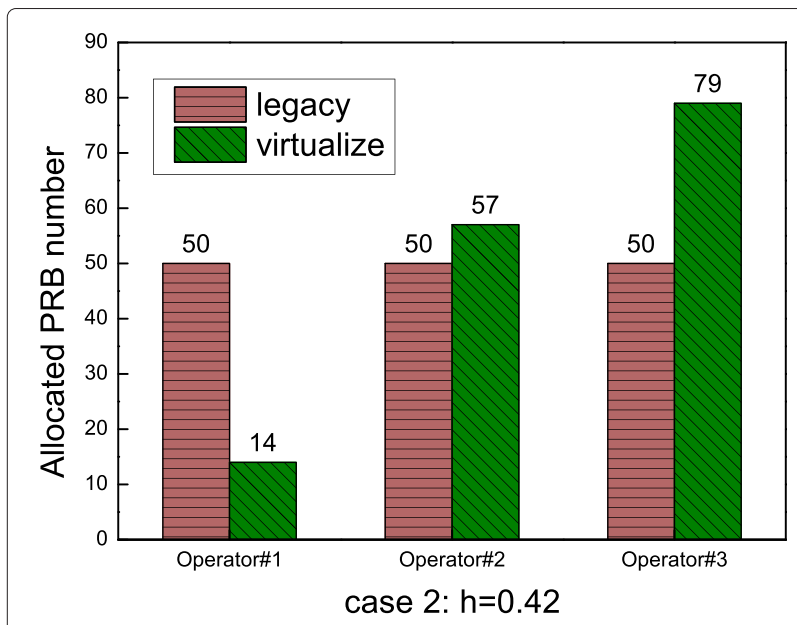

Fig. 4 Operator PRB allocation of case 2

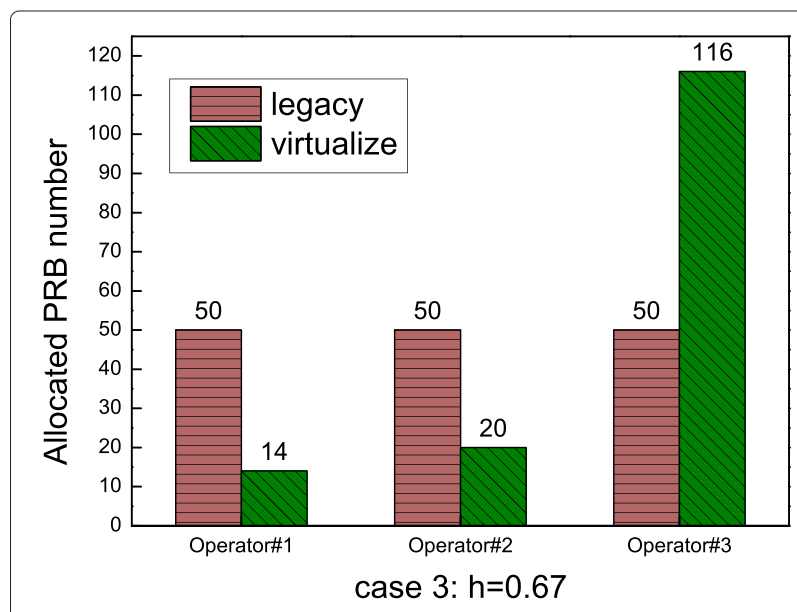

Fig. 5 Operator PRB allocation of case 3

and constraints. Case 1 exhibits ideally identical traffic rate while in case 2 VOs undertake disequilibrium traffic requirements. Case 3 further increases the traffic rate disequilibrium, where the dominated $\mathrm{VO} \# 3$ generate more than $90 \%$ of the total rate. Correspondingly, user behavior coefficient $h$ takes $0,0.42$, and 0.67. And different $h$ values can effectively reflect different VO traffic condition. Through Figs. 3, 4 and 5, it is observed that the proposed PRB allocation enables multiple VOs to dynamically share the PRBs according to different traffic conditions. By contrast, the static PRB management in legacy LTE network is rather ossified.

Figure 6 compares operators' average EE in kbits/Joule. In vertical, the proposed allocation scheme outperforms the legacy network setup. This is caused by the multiplexing gain from virtualization, i.e., the additional flexiblity provided to VOs of scheduling PRBs from the shared resource pool (possibly with better channel condition). In horizontal, EE of legacy network decreases as disequilibrium increases. This is because the redundant PRBs in the

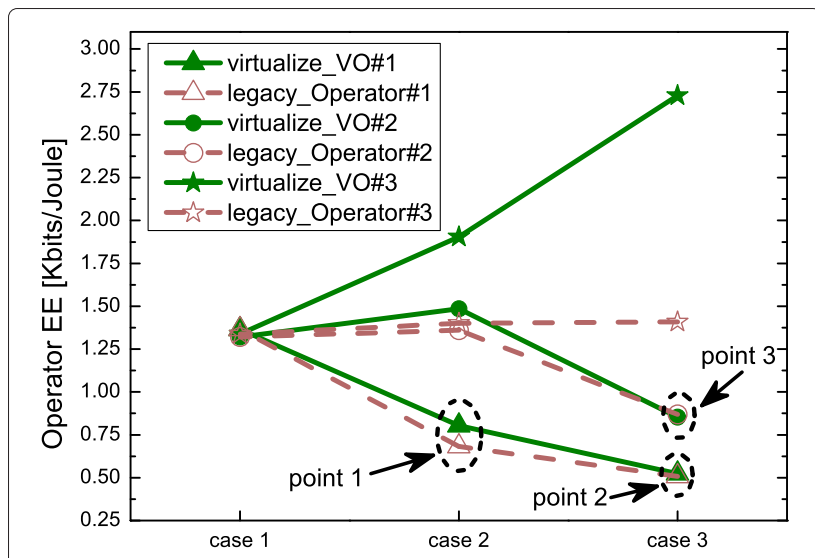

Fig. 6 Operator EE of simulation cases 1-3 
legacy network are wasted when traffic rate is satisfied or in call silence. By contrast, virtualization enables the redundant PRBs to be used by VO\#3 with comparatively heavy traffic load.

From the above analysis, we can conclude that VO\#1 is "economical" because of its light traffic burden. By contrast, "greedy" VO\#3 claims high-rate services and consumes a large amount of resources. While VO\#2 is rather "elastic," for the reason that it expands or shrinks its service level by dynamically obtaining access to mobile resource from the shared pool. This is the so-called flexibility provided by the virtualization, which greatly improves the system efficiency.

To further investigate the multiplexing gain brought about by virtualization, we demonstrate operator EE in Fig. 7 with configurable BS bandwidth. Figure 7 shows EE of operator\#3 by changing user behavior coefficient $h$ and BS bandwidth. The reason we select operator\#3 is that the performance of such resource-consuming operator is notably enhanced through the proposed virtualization in our work. As indicated in Fig. 7, EE gain in point 1 , point 2 , and point 3 are $0.068,1.43$, and 1.08 (kbits/Joule), respectively. Compared point 2 with point 3 , the EE gain is improved $33.80 \%$ when system overall bandwidth increases from 20 to $30 \mathrm{MHz}$. Compared point 2 to point 1 , the EE gain improves from 0.072 to 1.47 when $h$ increases from 0 to 0.67 . This reveals that the proposed virtualization approach can be even more advantageous with large-scaled bandwidth and traffic configuration.

Figure 8 demonstrates the cumulative distribution function (CDF) of system EE. Compared with legacy network setup, overall system EE is improved $23.25 \%$ on average.

Figure 9 shows the convergence of Algorithm 1. It can be observed that the method has a fast convergence rate; it converges to $90 \%$ of the upper bound performance within five iterations.

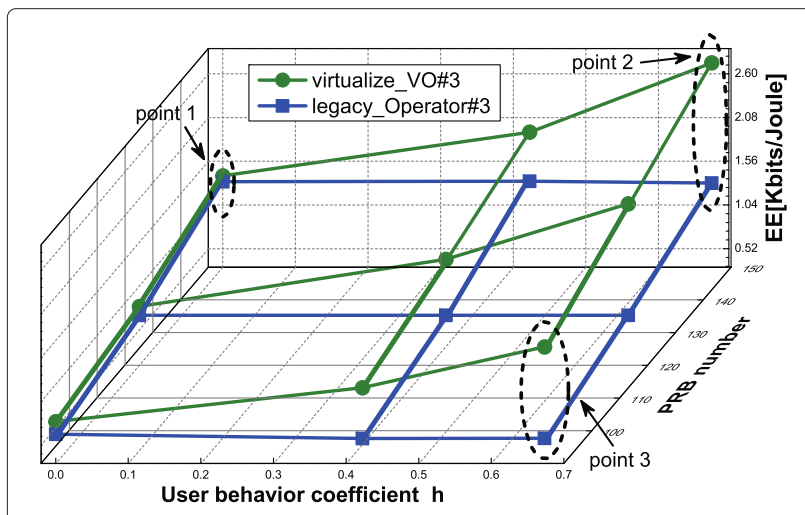

Fig. 7 Multiplexing gain from virtualization represented by increasing user behavior coefficient and BS bandwidth

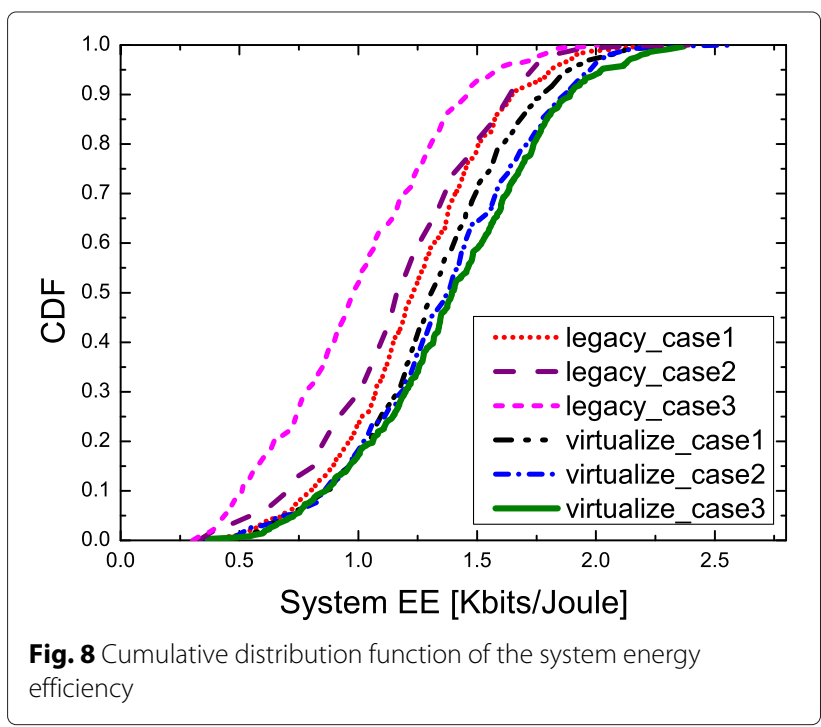

\section{Conclusions}

In this paper, a novel LTE air interface virtualization architecture is proposed based on user behavior analysis. An EE resource allocation problem is studied to support dynamic resource sharing among multiple VOs. We formulate the problem as a mixed combinatorial and non-convex optimization problem. To reduce the computational complexity, the problem is decoupled into two steps. First, to guarantee fairness, bankruptcy game and Shapley value are introduced to develop a heuristic bandwidth allocation problem. Second, fractional programming and convex optimization are adopted to obtain the optimal power allocation.

In simulation, PRBs are dynamically allocated to multiple VOs based on different user traffic demands. The defined user behavior can effectively reflect the VO traffic condition. The flexibility is proved in the sense that

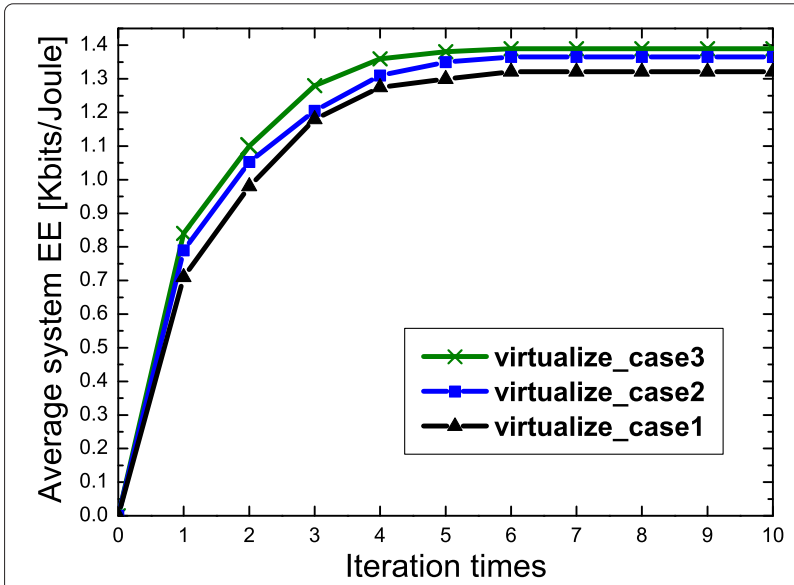

Fig. 9 Convergence of Algorithm 1 
rate-consuming VOs can get more PRB resource while a minimum number of PRBs are reserved for "economical" VOs to maintain the network operated. Besides, energy efficiency is improved in comparison with conventional networks.

\section{Appendix 1}

\section{Proof of Theorem 1}

First, let $\mathbf{P}^{*}$ be the solution of problem (16). We have:

$$
q^{*}=R_{\text {tot }}\left(\mathbf{P}^{*}\right) / P_{\text {tot }}\left(\mathbf{P}^{*}\right) \geq R_{\text {tot }}(\mathbf{P}) / P_{\text {tot }}(\mathbf{P})
$$

Hence:

$$
\begin{aligned}
& R_{\text {tot }}(\mathbf{P})-q^{*} P_{\text {tot }}(\mathbf{P}) \leq 0 \\
& R_{\text {tot }}\left(\mathbf{P}^{*}\right)-q^{*} P_{\text {tot }}\left(\mathbf{P}^{*}\right)=0
\end{aligned}
$$

From (a), we know that $\max \left\{R_{\text {tot }}(\mathbf{P})-q^{*} P_{\text {tot }}(\mathbf{P})\right\}=0$. From (b), we know that the maximum is taken on at point $\mathbf{P}^{*}$. Thus, the first part of our proof is finished.

Second, let $\mathbf{P}^{*}$ be the solution of problem (17). Based on the definition of (17), we can obtain following equations:

$$
R_{\text {tot }}(\mathbf{P})-q^{*} P_{\text {tot }}(\mathbf{P}) \leq R_{\text {tot }}\left(\mathbf{P}^{*}\right)-q^{*} P_{\text {tot }}\left(\mathbf{P}^{*}\right)=0
$$

Hence:

$$
\begin{aligned}
& R_{\text {tot }}(\mathbf{P})-q^{*} P_{\text {tot }}(\mathbf{P}) \leq 0 \\
& R_{\text {tot }}\left(\mathbf{P}^{*}\right)-q^{*} P_{\text {tot }}\left(\mathbf{P}^{*}\right)=0
\end{aligned}
$$

From (a), we have $q^{*} \geq R_{\text {tot }}(\mathbf{P}) / P_{\text {tot }}(\mathbf{P})$, i.e., $q^{*}$ is the maximum value of problem (16). From (b), we have $q^{*}=$ $R_{\text {tot }}\left(\mathbf{P}^{*}\right) / P_{\text {tot }}\left(\mathbf{P}^{*}\right)$, i.e., $\mathbf{P}^{*}$ is the solution vector of problem (16). Thus, the equivalence of the two problems defined in Theorem 1 is proved.

\section{Appendix 2}

\section{Proof of Algorithm 2 convergence}

Note that the problem equivalent to $(17)$ is $F(q)=$ $\max _{\mathbf{P}}\left\{R_{\text {tot }}(\mathbf{P})-q P_{\text {tot }}(\mathbf{P})\right\}$, as defined in Theorem 1. And the proof can be decoupled into three steps as follows:

\section{Step 1}

$\mathrm{F}(q)$ is a nonnegative function in the definition domain.

Proof. Assuming that $\mathbf{P}$ is an arbitrary solution of the problem and $q=R_{\text {tot }}(\mathbf{P}) / P_{\text {tot }}(\mathbf{P})$. Hence:

$$
\begin{aligned}
\mathrm{F}(q) & =\max _{\mathbf{P}}\left\{R_{t o t}(\mathbf{P})-q P_{t o t}(\mathbf{P})\right\} \\
& \geq R_{\text {tot }}(\mathbf{P})-q P_{\text {tot }}(\mathbf{P})=0
\end{aligned}
$$

\section{Step 2}

$\mathrm{F}(q)$ is a strictly monotonic decreasing function with respect to $q$.
Proof. Assuming $\mathbf{P}_{1}$ and $\mathbf{P}_{2}$ are two optimal solutions to $\mathrm{F}\left(q_{1}\right)$ and $\mathrm{F}\left(q_{2}\right)$, respectively, and $q_{1}<q_{2}$. Hence,

$$
\begin{aligned}
F\left(q_{2}\right) & =\max _{\mathbf{P}}\left\{R_{\text {tot }}(\mathbf{P})-q_{2} P_{\text {tot }}(\mathbf{P})\right\} \\
& =R_{\text {tot }}\left(\mathbf{P}_{2}\right)-q_{2} P_{\text {tot }}\left(\mathbf{P}_{2}\right) \\
& <R_{\text {tot }}\left(\mathbf{P}_{2}\right)-q_{1} P_{\text {tot }}\left(\mathbf{P}_{2}\right) \\
& \leq \max _{\mathbf{P}}\left\{R_{\text {tot }}(\mathbf{P})-q_{1} P_{\text {tot }}(\mathbf{P})\right\}=F\left(q_{1}\right)
\end{aligned}
$$

As for any arbitrary pair $q_{1}<q_{2}$, we have $\mathrm{F}\left(q_{1}\right)>\mathrm{F}\left(q_{2}\right)$.

Step 3

$q_{l}>q_{l+1}, \forall l=1,2, \ldots$ in Algorithm 2.

Proof. According to Algorithm 2 definition, $\mathbf{P}_{l}$ is the optimal solution to $\mathrm{F}\left(q_{l}\right)$ in $l$-th iteration and $q_{l+1}=$ $R_{\text {tot }}\left(\mathbf{P}_{l}\right) / P_{\text {tot }}\left(\mathbf{P}_{l}\right)$. Based on step 1, $F\left(q_{l}\right)>0$, the following fact is obtained:

$$
\begin{aligned}
F\left(q_{l}\right) & =R_{\mathrm{tot}}\left(\mathbf{P}_{l}\right)-q_{l} P_{\mathrm{tot}}\left(\mathbf{P}_{l}\right) \\
& =R_{\mathrm{tot}}\left(\mathbf{P}_{l}\right)\left(q_{l+1}-q_{l}\right)>0
\end{aligned}
$$

Since $R_{\text {tot }}\left(\mathbf{P}_{l}\right)>0$, we have $q_{l+1}>q_{l}$.

Based on steps $1-2$, we know that $\mathrm{F}(q)$ is a strictly monotonic decreasing function with nonnegative value. According to step 3, $q_{l}$ increases as iteration number $l$ accumulates, i.e., $q_{l+1}>q_{l}$. Therefore, as long as the iteration time is large enough, this will eventually approach to zero and satisfy the optimality condition of Theorem 1 , i.e., $l \rightarrow \infty, F\left(q_{l}\right) \rightarrow 0$.

\section{Competing interests}

The authors declare that they have no competing interests.

\section{Acknowledgements}

This work was supported by the National Natural Science Foundation of China (No. 61471060) and Funds for Creative Research Groups of China (No. 61421061).

\section{Author details}

${ }^{1}$ State Key Laboratory of Networking and Switching Technology, Beijing University of Posts and Telecommunications, Beijing, China. ${ }^{2}$ ZTE Corporation, Shenzhen, China.

Received: 20 October 2015 Accepted: 6 April 2016

Published online: 18 April 2016

\section{References}

1. Y Xu, J Wang, Q Wu, Z Du, L Shen, A Anpalagan, A game-theoretic perspective on self-organizing optimization for cognitive small cells. Commun Mag. IEEE. 53(7), 100-108 (2015)

2. Cisco, Global Mobile Data Traffic Forecast Update 2010-2015 White Paper (2011)

3. H Wen, PK Tiwary, T Le-Ngoc, Wireless virtualization. (Springer-Verlag, New York, NY, USA, 2013)

4. L Chengchao, FR Yu, Wireless network virtualization: a survey, some research issues and challenges. Commun. Surv. Tutorials IEEE. 17, 358-380 (2015)

5. A Gudipati, D Perry, LE Li, S Katti, in Proc. ACM SIGCOMM Workshop HotSDN. Softran: Software defined radio access network (ACM, Hong Kong, China, 2013), pp. 25-30 
6. LE Li, ZM Mao, J Rexford, Cellsdn: software-defined cellular networks, Princeton Univ.Press, Princeton, NJ, USA. Tech. Rep. TR- 922-12 Computer Science (2012)

7. M Yang, et al, in Proc. ACM SIGCOMM. OpenRAN: A software-defined RAN architecture via virtualization, (Hong Kong, China, 2013), pp. 549-550

8. Y Zaki, L Zhao, C Goerg, A Timm-Giel, LTE mobile network virtualization. Mobile Netw Appl. 16(4), 424-432 (2011)

9. L Zhao, M Li, Y Zaki, A Timm-Giel, C Gorg, LTE virtualization: from theoretical gain to practical solution, (San Francisco, CA, USA, 2011), pp. 71-78

10. Y Zaki, LTE optimization and mobile network virtualization, (Springer-Verlag, New York, NY, USA, 2012)

11. X Costa-Perez, J Swetina, G Tao, R Mahindra, S Rangarajan, Radio access network virtualization for future mobile carrier networks. Commun Mag. IEEE. 51, 27-35 (2013)

12. F Fangwen, UC Kozat, Stochastic game for wireless network virtualization. Netw IEEE/ACM Trans. 21, 84-97 (2013)

13. L Bin, T Hui, A bankruptcy game-based resource allocation approach among virtual mobile operators. IEEE Commun. Lett. 17(7), 1420-1423 (2013)

14. F Bo, T Hui, L Bin, in IEEE Proceedings of WCNC 2015 IEEE Wireless Communications and Networking Conference. Game theory based power allocation in LTE air interface virtualization (IEEE, New Orleans, LA USA, 2015), pp. 972-976

15. Z Zaidi, V Friderikos, MA Imran, Future RAN architecture: SD-RAN through a general-purpose processing platform. Vehicular Technol Mag. IEEE. 10, 52-60 (2015)

16. A Capone, I Filippini, B Gloss, U Barth, in IEEE Proceedings of the Second IFIP Conference on Sustainable Internet and ICT for Sustainability. Rethinking cellular system architecture for breaking current energy efficiency limits (IEEE, Pisa, Italy, 2012), pp. 1-5

17. A Osseiran, F Boccardi, V Braun, K Kusume, P Marsch, M Maternia, et al, Scenarios for $5 \mathrm{G}$ mobile and wireless communications: the vision of the METIS project. Commun. Mag. IEEE. 52, 26-35 (2014)

18. Z Xing, Y Rong, Z Yan, G Yue, M Im, L Cuthbert, et al, Energy-efficient multimedia transmissions through base station cooperation over heterogeneous cellular networks exploiting user behavior. Wireless Commun. IEEE. 21, 54-61 (2014)

19. Z Xing, Z Yan, Y Rong, W Wenbo, M Guizani, Enhancing spectral-energy efficiency for LTE-advanced heterogeneous networks: a users social pattern perspective. Wireless Commun. IEEE. 21, 10-17 (2014)

20. H Yu, Z Xing, Z Jiaxin, T Jian, S Zhuowen, W Wenbo, Energy-efficient design in heterogeneous cellular networks based on large-scale user behavior constraints. Wireless Commun. IEEE Trans. 13, 4746-4757 (2014)

21. C Gini, "Italian: Variabilità e mutabilità" Variability and Mutability', C. Cuppini, Bologna, 156 pages. Reprinted in Memorie di metodologica statistica. (E Pizetti, T Salvemini, eds.), (Rome: Libreria Eredi Virgilio Veschi (1955), 1912). https://en.wikipedia.org/wiki/Gini_coefficient

22. M Hazewinkel (ed.), Multinomial coefficient, Encyclopedia of Mathematics (Springer, 2001). http://en.wikipedia.org/wiki/Multinomialtheorem

23. Y Chen, S Zhang, S Xu, G Li, Fundamental trade-offs on green wireless networks. IEEE Commun. Mag. 49(6), 30-37 (2011)

24. M Pulido, JS Soriano, N Llorca, Game theory techniques for university management: an extended bankruptcy model. Oper Res. 109, 129-142 (2002)

25. C Georgios, E Edith, W Michael, Cooperative game theory: basic concepts and computational challenges. IEEE Intell. Syst. 27(3), 86-90 (2012)

26. W Dinkelbach, On nonlinear fractional programming. Manage Sci. 13(7), 492-498 (1967)

27. CIsheden, Z Chong, EA Jorswieck, G Fettweis, Framework for link-level energy efficiency optimization with informed transmitter. IEEE Trans. Wireless Commun. 11(8), 2946-2957 (2012)

28. S Boyd, L Vandenberghe, Convex optimization. (Cambridge Univ. Press, Cambridge, UK, 2004)

29. G Piro, L Grieco, G Boggia, F Capozzi, P Camarda, Simulating LTE cellular system: an open source framework. IEEE Trans, Veh. Technol. 60(2), 498-513 (2010)

30. E Westman, Calibration and evaluation of the exponential effective SINR mapping (EESM) in 802.16. Master Thesis, Stockholm, Sweden (2006)

\section{Submit your manuscript to a SpringerOpen ${ }^{\circ}$ journal and benefit from:}

- Convenient online submission

- Rigorous peer review

- Immediate publication on acceptance

- Open access: articles freely available online

- High visibility within the field

- Retaining the copyright to your article

Submit your next manuscript at $\gg$ springeropen.com 